University of Nebraska - Lincoln

DigitalCommons@University of Nebraska - Lincoln

Final Reports \& Technical Briefs from Mid-

America Transportation Center

Mid-America Transportation Center

2019

\title{
Validation of the Highway Capacity Manual Urban Street Travel Time Reliability Methodology using Empirical Data
}

\author{
Ernest Tufuor \\ University of Nebraska - Lincoln, ernest.tufuor@huskers.unl.edu \\ Laurence Rilett \\ University of Nebraska - Lincoln, Irilett2@unl.edu
}

Follow this and additional works at: https://digitalcommons.unl.edu/matcreports

Part of the Civil Engineering Commons, and the Transportation Engineering Commons

Tufuor, Ernest and Rilett, Laurence, "Validation of the Highway Capacity Manual Urban Street Travel Time Reliability Methodology using Empirical Data" (2019). Final Reports \& Technical Briefs from Mid-America Transportation Center. 99.

https://digitalcommons.unl.edu/matcreports/99

This Article is brought to you for free and open access by the Mid-America Transportation Center at DigitalCommons@University of Nebraska - Lincoln. It has been accepted for inclusion in Final Reports \& Technical Briefs from Mid-America Transportation Center by an authorized administrator of DigitalCommons@University of Nebraska - Lincoln. 


\title{
Validation of the Highway Capacity Manual Urban Street Travel Time Reliability Methodology using Empirical Data
}

\author{
Ernest O. A. Tufuor ${ }^{1}$ and Laurence R. Rilett ${ }^{2}$ \\ 1 University of Nebraska-Lincoln, Lincoln, NE \\ 2 Nebraska Transportation Center, University of Nebraska-Lincoln, Lincoln, NE \\ Corresponding author — Ernest O. A. Tufuor, ntc-etufuor@unl.edu
}

\begin{abstract}
The 6th edition of the Highway Capacity Manual (HCM-6) includes the concept of travel time reliability (TTR), which attempts to determine the distribution of average trip travel times over an extended period. TTR is an inherent part of travelers' route choice decisions and is used by traffic managers to better quantify operations rather than simply using average travel times. The focus of this paper is on the HCM-6 urban street TTR methodology contained in Chapter 17. The approach uses historical data (e.g., weather and volume fluctuations) and simple empirical data (e.g., 1-day volume count) to provide the user with average travel time and reliability predictions for a traffic facility over an extended period (e.g., a year). To the best of the authors' knowledge, there is no existing literature on validating the HCM-6 methodology with empirical data. The goals of this paper were to validate the HCM- 6 urban street reliability methodology by comparing the empirical Bluetooth (BT) travel time distributions with the estimated HCM-6 distribution, and to
\end{abstract}

Published in Transportation Research Record, Vol. 2673, no. 4 (2019), pp 415-426. doi 10.1177/0361198119838854

Copyright (c) 2019 National Academy of Sciences: Transportation Research Board; published by SAGE Publications. Used by permission. 
propose potential HCM- 6 augmentation strategies. Archived BT data from a 0.5mi urban arterial in Lincoln, Nebraska was used for comparison. It was found that there were statistically significant differences, but minimal practical differences, between the mean of the predicted HCM- 6 travel time distribution and the mean of the empirical distribution. However, the HCM- 6 distribution had a lower variance than the empirical distribution. Not surprisingly, the HCM- 6 model underestimated the TTR metrics (buffer index and the planning time index) by approximately $62 \%$ and $9 \%$, respectively.

Traffic agencies use travel time distributions for many applications including improving (a) transportation agency operations, (b) information for travelers' trip planning purposes, (c) arterial and freeway rerouting and detours selections, (d) calibration and validation of traffic microsimulation models, and travel time reliability (TTR) metrics. The benefits of accurate reporting of travel times are presented by Toppen and Wunderlich (1).

TTR has been defined in a number of different ways. For example, the United States Federal Highway Administration formally defines TTR "as the consistency or dependability in travel times, as measured from day-to-day and across different times of the day" (2). The Future Strategic Highway Research Program (F-SHRP) defines TTR as the variation in travel times over a time period, for example, an hour-to-hour or dayto-day variations (3). Other SHRP projects used the concept of variability to define TTR. A broad definition was proposed by the SHRP report 2 project L08 as follows:

TTR aims to quantify the variation of travel time. It is defined using the entire range of travel times for a given trip, for a selected time period (e.g., the P.M. peak hour during weekdays) over a selected horizon (e.g., a year). For the purpose of measuring reliability, a trip can be defined as occurring on a specific segment, facility (combination of multiple consecutive segments), or any subset of the transportation network, or the definition can be broadened to include a traveler's initial origin and final destination. Measuring travel time reliability requires that a sufficient history is described by the travel time distribution for a given trip.

It is clear that an accurate travel time distribution is a critical input for successful TTR applications.

The current and 6th edition of the Highway Capacity Manual (HCM6) assumes that TTR reflects "the distribution of trip travel time over an 
extended period" (4) and uses travel time distributions as input for deriving the TTR performance metrics of a road segment or facility. The HCM-6 identifies and defines two groups of TTR performance metrics: (1) timebased (e.g., buffer time), and (2) index-based (e.g., travel time index).

Chapter 17 of the HCM-6 proposes a methodology for evaluating the TTR metrics that are experienced by motorists on an urban street facility, and this is the focus of this paper. The HCM- 6 approach uses historical data (e.g., weather and volume fluctuations) and simple empirical data (e.g., 1-day volume count) to provide the user with a predicted travel time distribution for a given extended period of time (e.g., 1 year). This distribution is used to identify (1) the expected average travel time and (2) the forecast reliability metrics for the extended period. Note that the HCM-6 approach does not separate the effect of measurement or prediction errors from the reliability estimates.

\section{Overview of HCM-6 Methodology}

The HCM- 6 methodology is designed to account for the main causes of travel time variability including (a) traffic demand variations, (b) incident occurrences, (c) weather events, (d) work zones, and (e) special events. The approach incorporates demand fluctuations and the nonrecurring congestion effects to estimate current reliability metrics and predict future facility reliability metrics over a given time period (5).

Every analysis period (e.g., 15-min interval) within the reliability reporting period (typically, 6 months to a year) is identified as a scenario. A unique combination of the main causes of variability is defined for each scenario. The effect of these factors on running speed or saturation flow rates is quantified for each scenario.

The scenarios are evaluated using an HCM- 6 computational engine to estimate the expected value of the performance measures for each arterial segment and the facility within an analysis period. The collective set of the performance (e.g., travel times) is used to formulate the travel time distribution for the reliability reporting period.

By definition, the resultant performance metric describes the variation in average travel time between the analysis periods or scenarios and not travel times from individual vehicles. Hence some of the variability in performance is not accounted for by the HCM- 6 methodology. Typically, a 15-min analysis period is used, and this was adopted in this paper as well.

It is important to note that the HCM-6 approach is based on a single day volume count within the year, and the volume for the remaining 364 days are estimated based on this volume, demand factors, predicted 
weather, and expected incident occurrence. There is no attempt "to isolate the effects of measurement error or prediction error from the reliability measurements or estimates" (5).

In addition, "when dealing with predictions of performance, no attempt was made to add a separate component for prediction uncertainty" (5). Therefore, the HCM-6 measurement of reliability includes measurement uncertainty, which is defined as an estimated amount by which an observed value may differ from the "true value."

The HCM- 6 methodology has default values for a number of inputs including demand factors, weather, and incidents. However, HCM-6 recommends the use of local datasets to minimize the uncertainties and the user may input this information into the $\mathrm{HCM}-6$ computational engine. Samandar et al. (6) validated the HCM-6 freeway TTR prediction using local probe data. It was shown that the HCM- 6 model could sufficiently predict travel times and reliability measures for freeways. However, to the best of the authors' knowledge, no existing literature has used real data to validate the HCM-6 urban street methodology. The goal of this paper is to fill the gap.

It is generally accepted that direct measurements of travel time are preferred over indirect travel time estimation methods such as estimating travel times from instantaneous speed collected at point detectors. Because Bluetooth (BT) data provide point-to-point travel time measurements at relatively inexpensive cost, these systems have been adopted across the United States.

It should be noted that with the advent of connected vehicles, Wi-Fi, sensors, and powerful communication devices, the ability to collect pointto-point travel time data will continue to improve.

The objective of this paper is to evaluate the TTR of an urban street facility using low cost archived BT data. The goals are to validate the HCM- 6 urban street reliability methodology by comparing an empirical BT average travel time distribution with the forecast $\mathrm{HCM}-6$ average travel time distribution, and to propose potential HCM- 6 augmentation strategies.

The remainder of this paper is organized as follows. First, the details of the selected study area are provided. This is followed by a brief description of the conceptualization of the HCM- 6 methodology and its application on the testbed. Next, the methodology for BT data collection and processing on the testbed are presented. Finally, a comparative analysis of the results of the BT and HCM- 6 outcomes is presented, and the proposed augmentation strategies of the HCM- 6 methodology are discussed. 


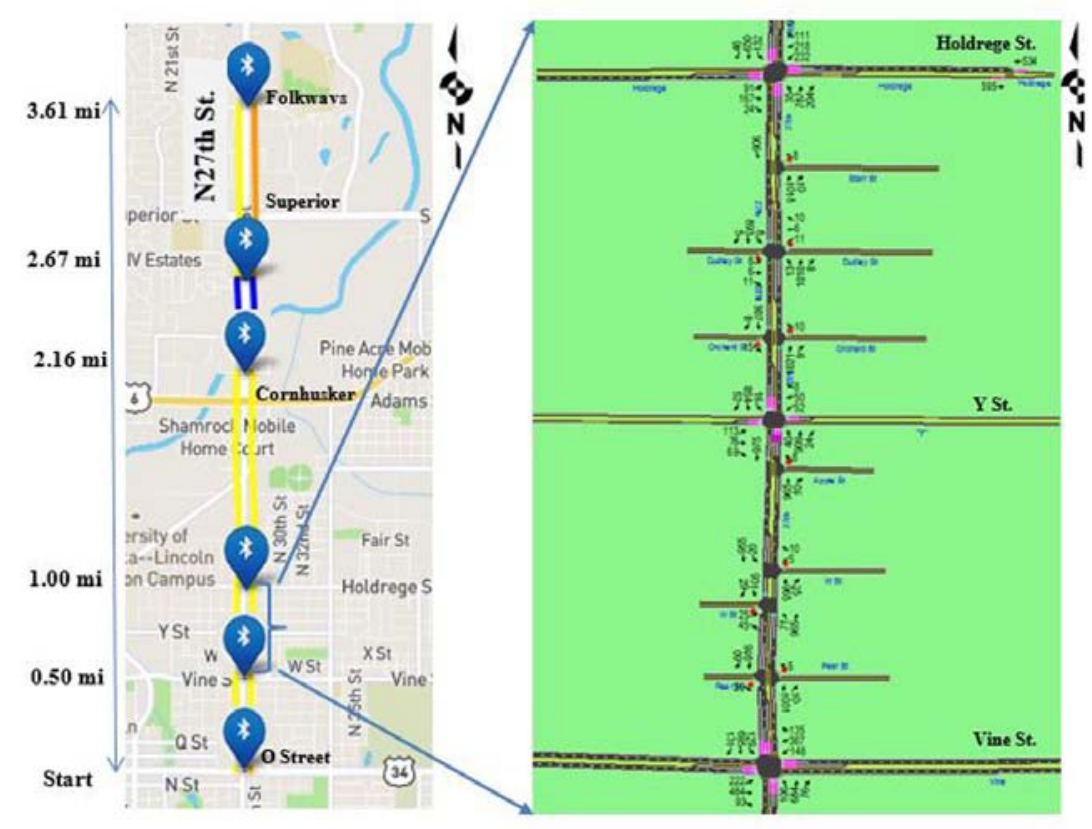

(a) Bluetooth detector locations

(b) HCM-6 testbed

Figure 1. Case study area showing $(\boldsymbol{a})$ Bluetooth detector locations and $(\boldsymbol{b})$ testbed volume count.

\section{Study Area, Data Collection, and Processing}

\section{Description of Study Area}

Figure 1 shows the 3.6-mi study corridor in Lincoln, NE. It is located on the N 27th Street from O Street in the south to Folkways Boulevard in the North. There are 14 signalized intersections, 32 exit/entry points per traffic direction, and the link distances vary from 0.5 to $1.2 \mathrm{mi}$. The selected HCM- 6 testbed, shown in Figure $1 b$, is $0.5 \mathrm{mi}$ in length and begins at Vine Street in the south and extends to Holdrege Street in the North. It has two lanes in both directions, a 1 to $2 \mathrm{~m}$ median, protected and permissible left turn movements, and a speed limit of 35 mph $(56.33 \mathrm{~km} / \mathrm{h})$.

The study corridor is part of the Nebraska Transportation Center's (NTC) arterial corridor system data collection and monitoring testbed.

The corridor was simulated using Synchro ${ }^{\mathrm{TM}}$, and Table 1 shows the relationship between signal delay and the estimated level of service (LOS) as a function of the period of day for each signalized intersection. 
Table 1. Study Corridor Signal Control Delay and Level of Service at Peak Periods

\begin{tabular}{|c|c|c|c|}
\hline \multirow{2}{*}{$\begin{array}{l}N 27^{\text {th }} \text { street signalized } \\
\text { intersections }\end{array}$} & \multicolumn{3}{|c|}{ Peak period signal delay (s) and LOS } \\
\hline & AM & Midday & $P M$ \\
\hline O St. & $58.7(\mathrm{E})$ & $41.1(D)$ & $48.4(D)$ \\
\hline P St. & $3.7(\mathrm{~A})$ & $7.2(\mathrm{~A})$ & $16.5(B)$ \\
\hline Vine St.* & $38.8(D)$ & $33.4(C)$ & $46.3(D)$ \\
\hline Y St.* & $13.9(B)$ & $10.2(B)$ & $20.0(B)$ \\
\hline Holdrege St. ${ }^{*}$ & $26.4(C)$ & $23.7(C)$ & $37.9(D)$ \\
\hline Fair St. & $5.2(\mathrm{~A})$ & $3.3(\mathrm{~A})$ & $4.6(\mathrm{~A})$ \\
\hline Cornhusker & $39.0(D)$ & $40.7(D)$ & $53.3(D)$ \\
\hline Knox St. & $9.0(\mathrm{~A})$ & $16.6(B)$ & $15.8(B)$ \\
\hline Fairfield St. & $10.0(\mathrm{~A})$ & $13.3(B)$ & $15.6(B)$ \\
\hline Old Dairy Rd. & $2.6(\mathrm{~A})$ & $4.7(\mathrm{~A})$ & $5.1(\mathrm{~A})$ \\
\hline Superior St. & $38.4(D)$ & 34.9 (C) & 38.7 (D) \\
\hline Ticonderoga & 10.9 (B) & $14.2(B)$ & $16.5(B)$ \\
\hline Kensington Dr. & $3.6(\mathrm{~A})$ & $8.7(\mathrm{~A})$ & $8.1(\mathrm{~A})$ \\
\hline Folkways Blvd. & $28.4(C)$ & $11.4(B)$ & $13.8(B)$ \\
\hline
\end{tabular}

*HCM testbed.

It may be seen in Table 1 that the estimated LOS on the corridor ranges from $A$ through $E$. The $\mathrm{HCM}-6$ testbed had control delays ranging from 10.2 to $46.3 \mathrm{~s}$, and corresponding LOS values that range from $B$ to $D$.

\section{Application of the HCM-6 Methodology}

\section{High-Level Representation}

The HCM-6 methodology for estimating the segment/ corridor averaged travel time distributions and the associated reliability performance metrics is illustrated in Figure 2.

The HCM-6 methodology assumes that the day-today variability in travel time can be estimated by adjusting demand and predicting weather and incident occurrences over an extended period of time. The method is based on a simulation approach in which prediction models on the impacts of changes in demand, incidents, and weather are used. The result is a forecast of the average travel time distribution over an extended period. This distribution is used to identify the average travel time on the corridor as well as key reliability metrics. 


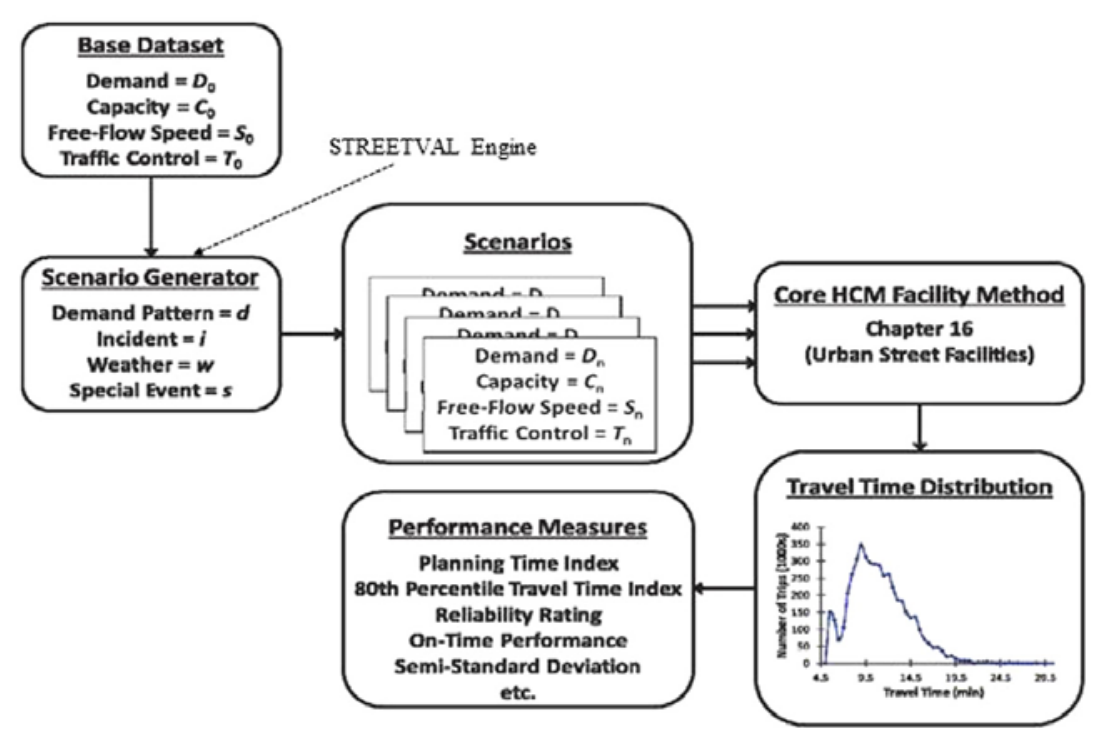

Figure 2. HCM-6 method for estimating average travel time distribution (Source: [4] Exhibit 17-2).

A brief description of the HCM-6 procedure is outlined as follows:

1. Create an input data file: This step requires the following data: (a) the functional class and geometric features of the subject urban street facility, (b) weather data [reference to NCDC (7)], (c) basic traffic volumes and count date and time under clear weather conditions, (d) segment and intersection annual crash frequency, and (e) duration of the analysis period (typically $15 \mathrm{~min}$ ), study period (e.g., PM peak 4.5 to $5.5 \mathrm{~h}$ ), and reliability reporting period (e.g., all weekdays in a year). The outcome of this step is a base dataset of the testbed, which consists of data required to evaluate the base performance for a single study period and data that describe weather, demand, and incident variations.

2. Scenario generation: This step involves the use of the HCM-6 computational engine, known as the STREET eVALuation (STREETVAL), to adjust the base dataset demand and capacity to reflect their variations under a combination of conditions. The engine has four steps that are conducted sequentially. First, the engine predicts weather event date, time, duration, and type. Secondly, it identifies traffic adjustment factors for time and date in the reporting period. Thirdly, it predicts incident event date, time, duration, event type (crash or no crash), crash severity level, and incident location. 
Lastly, the STREETVAL produces one input file for each analysis period (i.e., each scenario) of the reliability reporting period. Each input file contains the adjustments to saturation flows at intersections, running speed on the segment, and traffic demand variations.

3. Facility evaluation: In this step, each segment scenario input file from the previous step is subjected to the HCM- 6 urban street facility evaluation methodology (i.e., Chapter 16) to predict the average travel time on the testbed for each analysis period. Note that the estimate represents an average of the 15-min aggregated travel time for each analysis period.

4. Performance measure: In this final step, the estimated averages for each analysis period are compiled over the reliability reporting period into an average travel time distribution. The statistical description of the distribution and the base free-flow travel times are used to determine reliability performance metrics.

\section{HCM-6 Travel Time Reliability Analysis}

For illustrative purposes, the reliability of two testbed segments on the N 27th Street were used for the HCM-6 analysis: (a) from Vine Street to Y Street; (b) from Y Street to Holdrege Street. The period analyzed was the PM peak period (4:30-5:30 p.m.). All weekdays were selected as the study period, and 1 year was selected as the reliability reporting period. The input data for the HCM-6 analysis are shown below in Table 2.

Table 2. HCM-6 Methodology Input Data

\begin{tabular}{|c|c|c|}
\hline Input & lt used & Non-default value used \\
\hline \multicolumn{3}{|l|}{ Reliability methodology } \\
\hline Geometric design & $\square$ & $\begin{array}{l}\text { Each segment is } 0.25 \mathrm{mi} \text { (Segment } 1=\text { Vine }-\mathrm{Y}, \\
\text { and Segment } 2=\mathrm{Y} \text {-Holdrege) }\end{array}$ \\
\hline Functional class & $\square$ & Urban principal arterial \\
\hline Analysis period & $\square$ & 15 min for the facility \\
\hline Study period & $\square$ & 4:30-5:30 p.m. for all weekdays \\
\hline Reliability period & $\square$ & 1 year (2016) \\
\hline Signal timings & $\square$ & Provided by the City of Lincoln \\
\hline Alternative dataset & 凶 & No work zones considered \\
\hline $\begin{array}{l}\text { Weather data, demand ratios, and } \\
\text { factors influencing accident duration }\end{array}$ & 凶 & Selected HCM-6 default data for Lincoln, NE \\
\hline Segment and intersection crash data & $\square$ & Available locally \\
\hline Scenario evaluation interval & $\square$ & $\begin{array}{l}\text { Evaluate the scenarios of each day in chronologic } \\
\text { order (i.e., set to 1) }\end{array}$ \\
\hline
\end{tabular}


The functional class input of the testbed is used in the HCM-6 to determine the hour-of-day and month-of-year traffic adjustment factors, which are based on the findings from Hallenbeck et al. (8).

It is important to note that the STREETVAL has a database of longterm regional weather conditions with probabilities developed on 10 years of data for Lincoln, NE, and other cities. Consequently, it is assumed that there are no geographic or spatial transferability issues related to weather.

Figures $\mathbf{3}$ and $\mathbf{4}$ give a snapshot of the base data input from the STREETVAL computational engine for the first segment of the testbed.

(a)

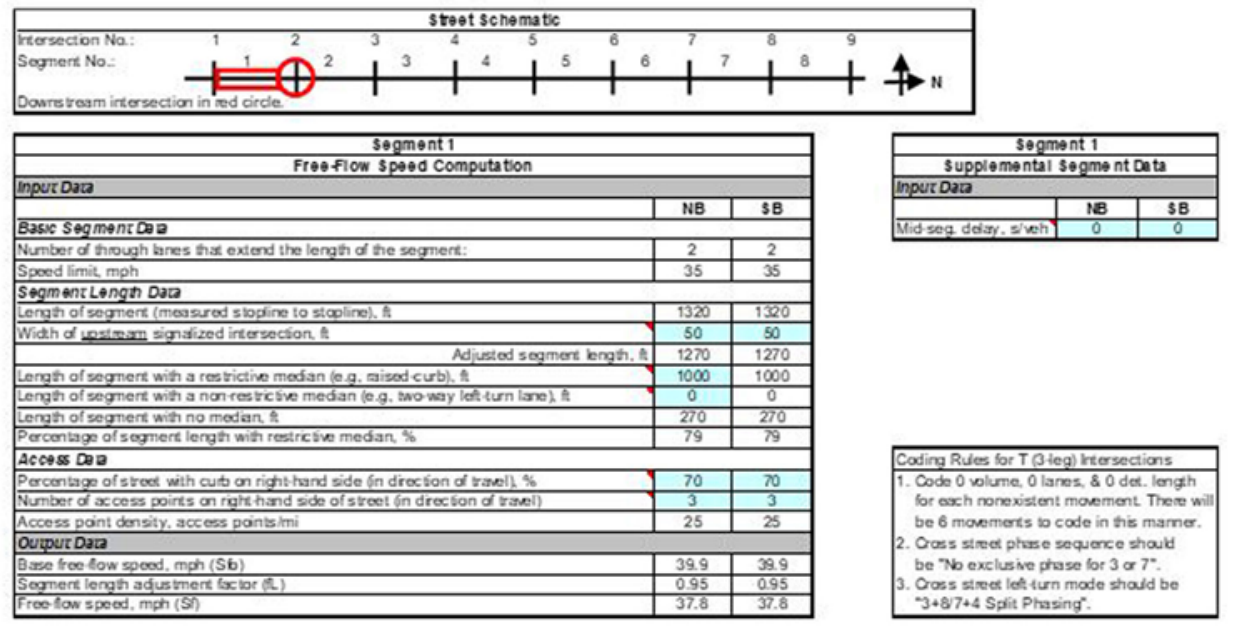

(b)



Figure 3. HCM-6 methodology computational engine: (a) free-flow speed computation; (b) segment and intersection details. 


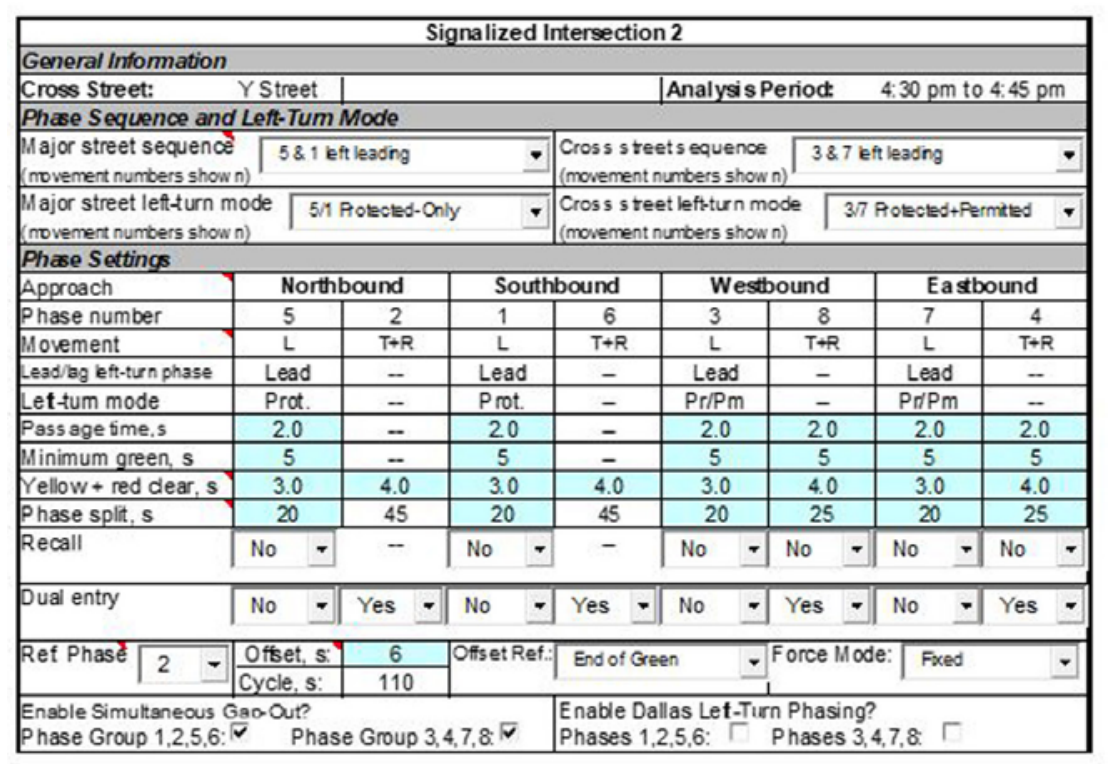

Figure 4. A typical intersection base dataset on the testbed.

The details of a typical intersection base dataset in the STREETVAL are presented in Figure 4. The signal timings are from the City of Lincoln; the PM peak hour settings were not changed over the reliability reporting period

Figure 5 provides the estimated average of the 15-min aggregates travel time distribution of each segment for the northbound movement (i.e., Segment A, from Vine Street to $Y$ Street) that was derived using the HCM-6 methodology.

It can be seen from Figure $5 a$ that the aggregated 15- min travel time distribution of Segment 1 is positively skewed with a long tail, in which the mean value is higher than the median value. Segment 2 (Figure 5b) has a bimodal distribution, which may be because of traffic signal coordination issues.

Following HCM-6 protocol, and since the HCM-6 methodology was based on a single day volume count within the year, and the volumes for the remaining 364 days were estimated, a simulation study was conducted on the travel time results to estimate the bounds.

Table 3 shows a summary of the predicted motorized vehicle performance metrics for the testbed after four repetitions. The simulation was done by varying demand and keeping weather, incidents, and all other factors constant. Given that for this study, the PM peak period is $1 \mathrm{~h}$, four 15-min analysis periods, and considering all weekdays in 2016 resulted in 4,176 separate estimates of average travel time. 
(a)

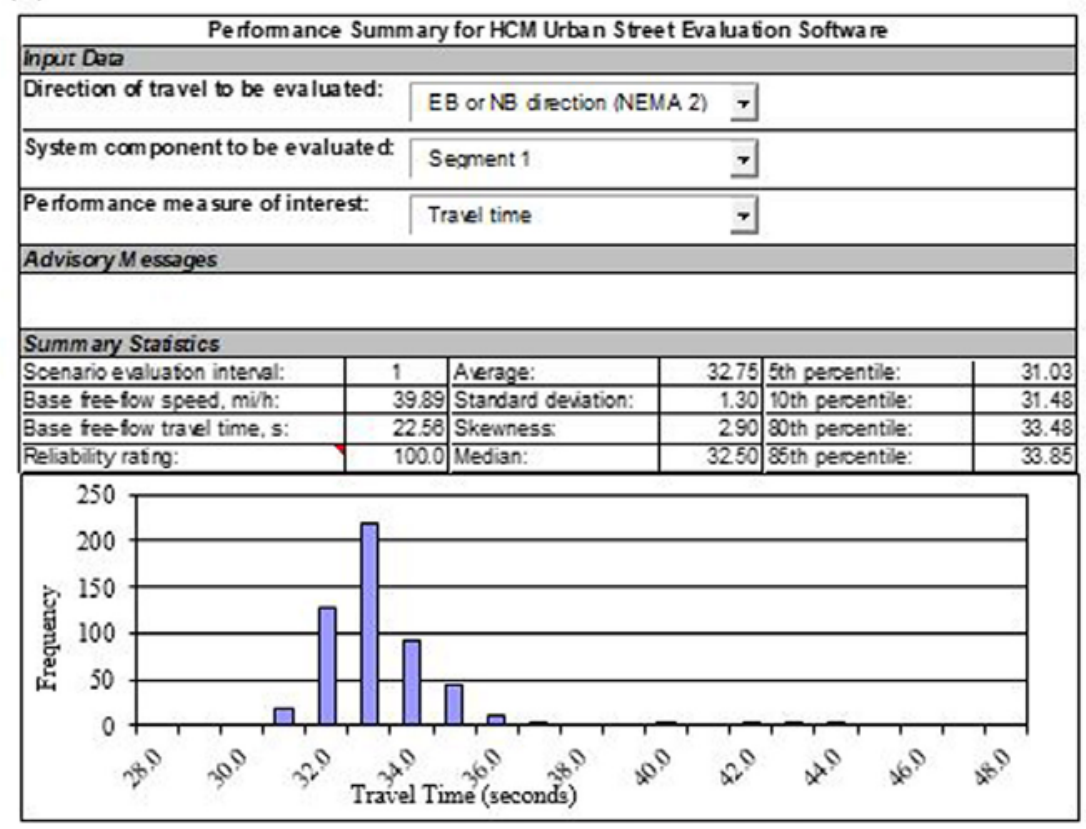

(b)



Figure 5. HCM-6 results for the averaged 15-min aggregate travel times on testbed: (a) Vine Street to Y Street; (b) Y Street to Holdrege Street. 
Table 3. HCM-6 Predicted Motorized Vehicle Performance Measures

\begin{tabular}{lrrr}
$\begin{array}{l}\text { Indicators (averaged 15-min } \\
\text { aggregates, } N=4,176)\end{array}$ & Mean & SD of mean & Median \\
\hline Travel time (s) & 69.59 & 3.50 & 68.94 \\
Travel speed (mph) & 25.93 & 1.26 & 26.11 \\
Stop rate (stops/mi) & 1.28 & 0.20 & 1.21 \\
Base free-flow travel time (s) & 45.17 & & \\
Level of service & $\mathrm{C}$ & & \\
$\quad$ (reference: Exhibit 16-3, HCM 2016) & & &
\end{tabular}

$\mathrm{SD}=$ standard deviation.

It may be seen from Table 3 that the HCM- 6 procedure forecast the average travel time of $69.59 \mathrm{~s}$ and an average speed of $26 \mathrm{mph}$, which is $65 \%$ of the free-flow speed. The vehicles stop at an average rate of 1.28 per mile, and the testbed operates at LOS C during the PM peak throughout the year.

In summary, the HCM-6 methodology forecasts average travel time and associated reliability metrics on urban arterials based on a single day volume input by a user. To the best of the authors' knowledge, there has been no research conducted to validate the HCM-6 urban street methodology using empirical data. In the following section, an empirical 15min average travel time distribution on the same HCM- 6 testbed over the same period of time is estimated using BT data.

\section{Application of the Empirical BT Travel Time Data}

\section{Data Collection}

Figure 6 illustrates the setup of the BT devices and the data collection system. The BT detectors are located in City of Lincoln traffic cabinets. The detectors capture the date and time of passive BT-enabled devices. The data are transmitted to a host computer server at NTC.

For security purposes, the detected unique media access control (MAC) addresses of the captured devices within the controller are encrypted and replaced with a new identifier (9). The encrypted data from all the testbed intersections are compared. When an exact match is found, the travel time is calculated by taking the difference in the timestamps. In this study, the link HCM-6 free-flow travel time was used as 


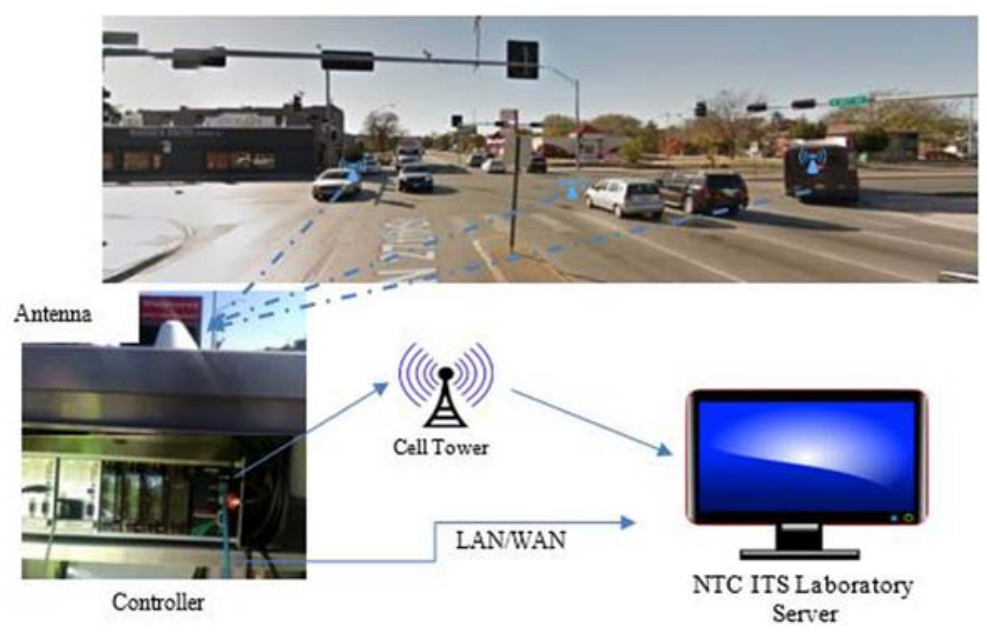

Figure 6. Bluetooth detector collection systems (Source: [9]).

the lower bound and twice the median BT travel time was used as the upper bound. Any travel time that was out of this range was considered an outlier and removed.

A total of 208,170 individual vehicle travel times were collected from January 2015 to May 2017 on the study corridor. The data were aggregated at 15-min intervals to ensure consistency with the HCM-6 methodology.

\section{Methodology for BT Data Processing}

The BT data processing methodology is presented in the flowchart shown in Figure 7. In the first step, the 15-min aggregates of the BT dataset are divided into a number of subsets. In this study, five subjects were identified. Each subset contains the empirical BT travel times corresponding to the time periods associated with each category. Note that these subsets are not mutually exclusive.

In the second step, a subset can be extracted from the BT dataset depending on the objectives of the user. For example, if the goal is to determine TTR for rainy days in AM peak periods, then the BT travel times for rainy days within AM peaks over the number of days for which reliability is to be computed (i.e., the reliability reporting period) is selected. In this study, the PM peaks for all weekdays was the scenario identified for analysis.

The third step is to determine the travel time distribution and descriptive statistics of the 15-min aggregates of the scenario under 




Figure 7. BT data processing methodology.

consideration. The distribution is used in the fourth step to determine the corresponding reliability performance metrics for the chosen scenario. Lastly, the above steps are performed for each road segment and aggregated to estimate the average 15-min travel time distribution on the corridor. The distribution is then used to estimate the TTR metrics.

\section{Bluetooth Data Analysis}

To use the BT dataset, it is necessary to test its capability and accuracy.

\section{BT Penetration Rate}

The BT penetration rate or sampling rate is defined as the ratio of the number of travel times captured by the BT sensor to the actual volume of arriving vehicles within a specific time frame. Its penetration rate for the $i^{\text {th }}$ intersection, $P_{i}$, can be mathematically expressed as:

$$
P_{i}=\frac{V_{B i}}{V_{A i}} \text { for all } i=1,2, \ldots, N \text { (the number of intersections) }
$$


where $V_{B i}=$ number of travel times captured by the BT sensor at the $i^{\text {th }}$ intersection and $V_{A i}=$ number of arriving vehicles at the $i^{\text {th }}$ intersection.

The penetration rate for each 15-min analysis period within the observed PM peak period (e.g., from 4:30-5:30 p.m.) was estimated using Equation 1.

The penetration rate ranged from $4 \%$ to $6 \%$ (for only a 2-day count), which is similar to estimated penetration rates from previous studies and is large enough to use for statistical inference (10-12). It should be noted that the rate does not account for double counting, (i.e., when two or more BT-enabled devices are captured from the same vehicle).

\section{BT Travel Time Trends}

Figure 8 illustrates the travel time trends for the $O$ Street to Vine Street segment of the five chosen subsets. Similar patterns were obtained from the other five segments but are not shown because of space constraints.
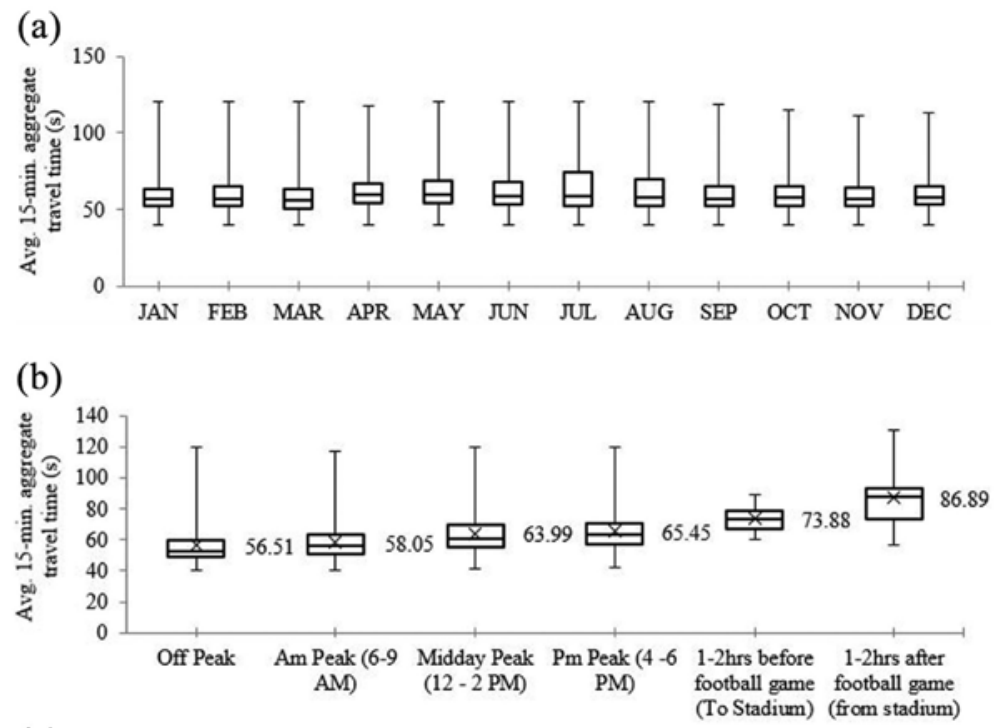

(c)

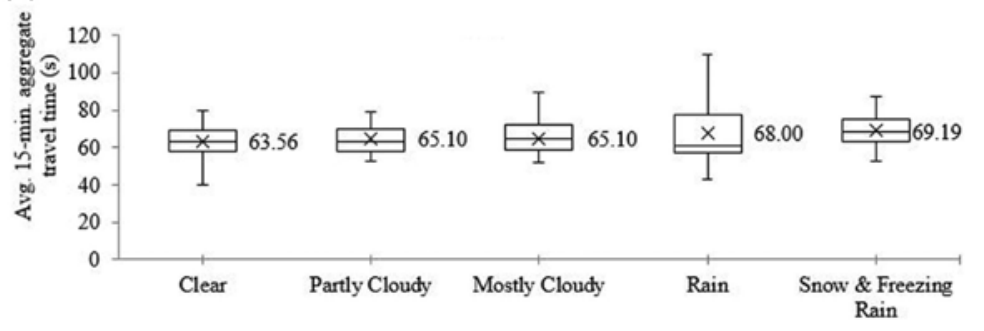

Figure 8. Box plots of Bluetooth travel times of $(\boldsymbol{a})$ monthly variations, $(\boldsymbol{b})$ traffic conditions, and (c) weather conditions from the O Street to Vine Street segment. 
The display format follows standard boxplot protocols where the bottom, the middle, and the top of each box represent the 25th percentile, the median, and the 75th percentile travel times, respectively. The points marked " $X$ " are the mean travel times and the maximum and minimum values are shown as the upper and lower limits of each box.

Figure $8 a$ shows the average 15 -min aggregate travel time pattern by month. It may be seen that, the summer months (June, July, August) generally have higher mean travel times and higher variability in travel time. It is hypothesized that this occurs because of increased construction.

Figure $8 b$ shows that the highest average 15-min aggregate travel times occur on football game days. This is not surprising because the football stadium, which has sold out for every game since 1962, hosts over 90,000 fans per game. On regular days, the PM peak has the highest travel times.

With regards to different weather conditions, Figure $8 c$ shows that rainy and snowy conditions have the highest travel times. This is not surprising because precipitation reduces tire friction, which results in drivers slowing down, increasing headways, and increasing travel times.

\section{BT Travel Time Distributions}

The descriptive statistics of each BT average 15-min aggregate travel time subsets, which correspond to Figure 8, are shown in Table 4.

It may be seen that, the mean of each subset is higher than its corresponding median value. Hence the distribution patterns are positively skewed and are consistent with the HCM-6 distribution in Figure 5.

The measure of dispersion (e.g., the standard deviation) are approximately 38\% larger in May-August as compared to other months. It is hypothesized that the difference in the dispersion may be because of the frequent stop and yield control within work zones that often occurs during summer.

The descriptive statistics support the findings in other empirical studies-that the distribution of link travel times is generally not symmetrical (13). Tufuor and Rilett give a more extensive statistical analysis to determine the best fit distributions for the data obtained on the same testbed (9). The lognormal and gamma distributions were found to fit best for the short and long links, respectively, as it also pertains in other studies $(10,13,14)$. 
Table 4. Descriptive Statistics of Averaged 15-min BT at Varying Conditions (s)

\begin{tabular}{|c|c|c|c|c|c|c|c|c|c|c|}
\hline Variability effects & Subsets & Min. & $\begin{array}{c}\text { First } \\
\text { quartile }\end{array}$ & Median & $\begin{array}{l}\text { Third } \\
\text { quartile }\end{array}$ & Max. & Mean & Size & $S D S$ & Skewness \\
\hline \multicolumn{11}{|c|}{ 1. Short arterial road links $(0.5 \mathrm{mi})$, that is, from $O$ Street to Vine Street } \\
\hline \multirow[t]{4}{*}{ Traffic condition } & Off-peak & 40.0 & 49.0 & 53.0 & 60.0 & 120.0 & 56.5 & 3,379 & 11.9 & 2.0 \\
\hline & AM peak & 40.0 & 51.0 & 56.0 & 63.0 & 117.0 & 58.1 & 3,263 & 10.7 & 1.4 \\
\hline & Midday peak & 41.0 & 55.0 & 61.0 & 70.0 & 120.0 & 64.0 & 2,413 & 12.4 & 1.5 \\
\hline & PM peak & 42.0 & 57.0 & 63.0 & 71.0 & 120.0 & 65.5 & 2,424 & 11.6 & 1.5 \\
\hline \multirow[t]{2}{*}{ Game day } & Pre-game & 60.0 & 67.0 & 73.5 & 79.0 & 89.0 & 73.9 & 26 & 8.0 & 0.2 \\
\hline & Post-game & 57.0 & 73.5 & 88.0 & 93.0 & 131.0 & 86.9 & 18 & 17.9 & 0.6 \\
\hline \multirow[t]{12}{*}{ Monthly variations } & Jan & 40.0 & 52.0 & 57.0 & 63.0 & 120.0 & 58.7 & 1,840 & 10.1 & 1.5 \\
\hline & Feb & 40.0 & 52.0 & 57.0 & 65.0 & 120.0 & 59.8 & 1,808 & 11.4 & 1.5 \\
\hline & Mar & 40.0 & 51.0 & 56.0 & 63.0 & 120.0 & 58.3 & 2,097 & 10.5 & 1.7 \\
\hline & Apr & 40.0 & 54.0 & 60.0 & 67.0 & 118.0 & 61.5 & 1,952 & 11.3 & 1.2 \\
\hline & May & 40.0 & 54.0 & 60.0 & 69.0 & 120.0 & 62.9 & 1,973 & 13.5 & 1.3 \\
\hline & Jun & 40.0 & 53.0 & 59.0 & 68.0 & 120.0 & 62.2 & 1,053 & 13.8 & 1.5 \\
\hline & Jul & 40.0 & 52.0 & 59.0 & 74.0 & 120.0 & 64.7 & 1,003 & 16.8 & 1.2 \\
\hline & Aug & 40.0 & 52.0 & 58.0 & 69.5 & 120.0 & 62.0 & 1,379 & 13.8 & 1.3 \\
\hline & Sept & 40.0 & 52.0 & 57.0 & 65.0 & 119.0 & 59.3 & 1,294 & 10.5 & 1.5 \\
\hline & Oct & 40.0 & 52.0 & 58.0 & 65.0 & 115.0 & 59.2 & 1,352 & 10.0 & 1.2 \\
\hline & Nov & 40.0 & 52.0 & 57.0 & 64.0 & 111.0 & 59.2 & 1,304 & 9.9 & 1.2 \\
\hline & Dec & 40.0 & 53.0 & 58.0 & 65.0 & 113.0 & 59.9 & 1,380 & 10.3 & 1.0 \\
\hline \multirow[t]{5}{*}{ Weather variations } & Clear & 40.0 & 58.0 & 63.0 & 69.0 & 80.0 & 63.6 & 299 & 7.7 & -0.2 \\
\hline & Snow & 58.0 & 63.5 & 68.5 & 75.3 & 84.0 & 69.2 & 32 & 7.6 & 0.5 \\
\hline & Rain & 50.0 & 57.0 & 61.0 & 77.5 & 95.0 & 68.0 & 15 & 15.4 & 0.8 \\
\hline & Partly cloudy & 44.0 & 58.3 & 63.0 & 70.0 & 102.0 & 65.1 & 42 & 11.7 & 0.9 \\
\hline & Mostly cloudy & 48.0 & 59.0 & 65.0 & 72.0 & 84.0 & 65.1 & 41 & 9.4 & 0.0 \\
\hline \multicolumn{11}{|c|}{ 2. Long arterial road links $(3.6 \mathrm{mi})$, that is, from O Street to Folkways } \\
\hline \multirow[t]{3}{*}{ Traffic condition } & AM peak & 311.0 & 419.0 & 464.0 & 513.0 & 555.0 & 467.9 & 1,558 & 63.0 & 0.06 \\
\hline & Midday peak & 311.0 & 419.8 & 466.0 & 514.0 & 576.0 & 530.4 & 1,404 & 62.9 & 0.06 \\
\hline & PM peak & 311.0 & 422.0 & 467.0 & 516.0 & 600.0 & 552.3 & 1,429 & 62.9 & 0.03 \\
\hline
\end{tabular}

$\mathrm{SD}=$ standard deviation .

\section{Comparative Analysis of Results}

Figure 9 shows the distributions of the averaged 15-min aggregated travel times and the corresponding cumulative frequency curves for both the HCM- 6 forecast average travel time distribution and the empirical average travel time distribution. 


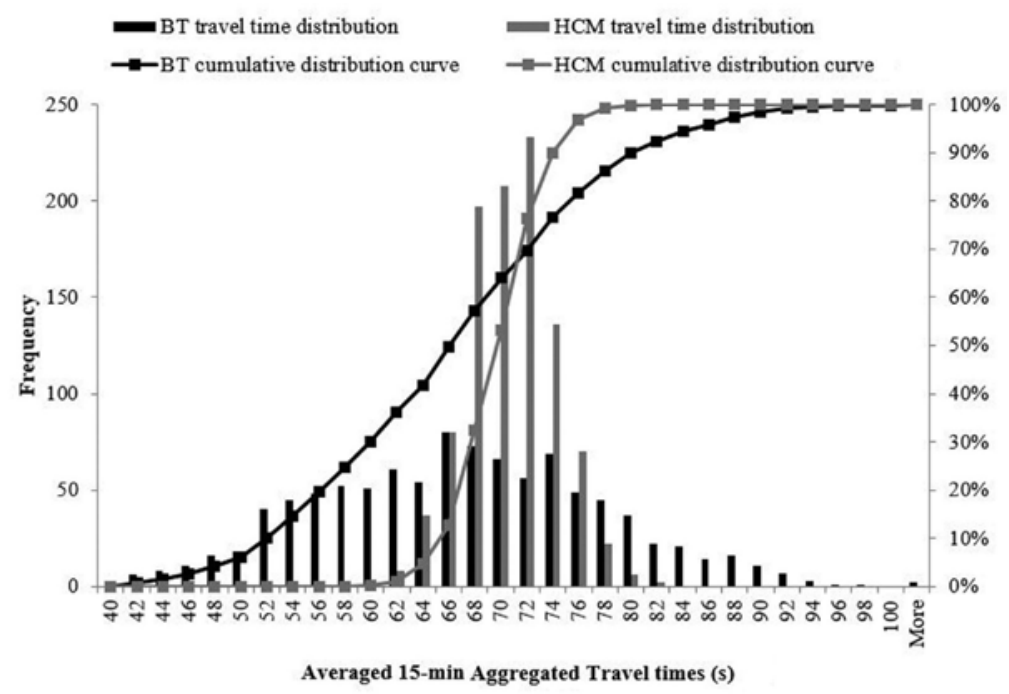

Figure 9. Travel time distributions and cumulative frequency curves.

It may be seen from Figure 9 that the mean travel times are relatively close. However, the forecast HCM- 6 model is much less dispersed as compared to the empirical travel time distribution. In fact, the range of the empirical distribution (52 s) is approximately three times greater than the range of the HCM- 6 distribution (18 s). The forecast HCM- 6 travel time distribution is less more dispersed as compared to the empirical BT travel time distribution.

Table $\mathbf{5}$ provides a summary of the results of the mean travel times and the reliability performance metrics for the weekday PM peak periods. It may be seen from Table 5 that there is a statistically significant difference, but not a practical difference, between the facility's mean travel times for the two procedures. The estimated mean travel from the HCM-6 is about $4 \mathrm{~s}$ higher than the BT case.

More critically, the variability of the BT travel time distribution is much higher than the HCM-6 travel time distribution. The following hypothesis was tested using analysis of variance, that is:

$$
\begin{aligned}
& \mathbf{H}_{\mathbf{0}} \text { : Population variance of HCM distribution= Population variance } \\
& \text { of empirical distribution } \\
& \mathbf{H}_{\mathrm{a}} \text { : Population variance of HCM distribution } \neq \text { Population variance } \\
& \text { of empirical distribution }
\end{aligned}
$$


Table 5. Testbed Reliability Performance and Statistics

\begin{tabular}{|c|c|c|c|}
\hline $\begin{array}{l}\text { Reliability performance measures of } \\
\text { average 15-min aggregates of travel time }\end{array}$ & $\begin{array}{r}H C M-6 \\
(S D)\end{array}$ & $\begin{array}{r}B T \\
(S D)\end{array}$ & $\begin{array}{l}\text { Difference } \\
\text { (\% change) }\end{array}$ \\
\hline Mean travel time (MTT) in seconds & $69.59(3.5)$ & $65.50(11.6)$ & $-4.1(-6)$ \\
\hline $95 \%$ confidence interval & {$[69.37,69.81]$} & {$[64.78,66.22]$} & \\
\hline $\begin{array}{l}\text { Mean travel time index (TTI) (ratio of } \\
\text { mean to free-flow travel time) }\end{array}$ & 1.54 & 1.46 & $-0.1(-5)$ \\
\hline $\begin{array}{l}\text { Planning time index (PTI) }\left(95^{\text {th }} \text { percentile }\right. \\
\text { time divided by the free-flow time) }\end{array}$ & 1.71 & 1.87 & $0.2(9)$ \\
\hline $\begin{array}{l}\text { Buffer index }\left(95^{\text {th }} \text { percentile travel time }\right. \\
\text { minus mean travel time, divided by } \\
\text { the mean travel time) }\end{array}$ & 0.11 & 0.29 & $0.2(62)$ \\
\hline $\begin{array}{l}t \text {-statistic (comparing mean travel times } \\
\text { assuming unequal variance) }\end{array}$ & \multicolumn{2}{|c|}{$9.70(p$-value $=1.1 \mathrm{E}-21)$} & \\
\hline F-statistic (analysis of variance ) & \multicolumn{3}{|c|}{$115.01(p$-value $=3.9 \mathrm{E}-26)$} \\
\hline
\end{tabular}

$\mathrm{SD}=$ standard deviation

The results show that there are statistically significant differences between the variance, and there is evidence to reject the null hypothesis. In other words, assuming a Gaussian distribution for both travel time distributions, the population variances are statistically different.

The travel time index (TTI) in both cases shows that the facility is not very congested. The percent of vehicle-miles-traveled (VMT) associated with a TTI less than 2.5 is more than $95 \%$. This implies that the facility is likely to provide more than 95\% of VMT associated with LOS D or better in both cases. That the testbed operates at LOS C during PM peak periods is confirmed in Table 4.

The planning time index of 1.71 for the HCM- 6 method implies that for a trip lasting $69.59 \mathrm{~s}$, one must plan a total time of about $119 \mathrm{~s}$. Whereas in the empirical BT case, one has to plan a total of $122 \mathrm{~s}$.

The buffer index depicts the extra time most travelers need to add to the average travel time to ensure on-time arrival. The HCM-6 underestimates the buffer index by about $62 \%$ as compared to the empirical $B T$ results.

\section{Potential HCM Augmentation Strategies}

This paper illustrated that the HCM-6 TTR methodology although useful and an important step for traffic operation analyses might not give 
accurate results. A number of potential reasons for this discrepancy were provided. For example, it is hypothesized that one of the leading causes is owing to the inability of the HCM-6 methodology to control both the systematic and random elements in the scenario generation process.

The systematic variations such as changes in weather and traffic demand on periods within the day, month, and year are recognized and predicted by the HCM- 6 using averages. It is also easy to hypothesize that the relative sparsity of the input data (e.g., 1-day volume data) and the reliance on default aggregate datasets (e.g., monthly weather information) may also result in forecast errors. This section discusses two potential augmentation strategies that the authors believe may lead to more accurate results.

\section{Calibrate the HCM-6 Model with Local Empirical Data}

At its heart, the HCM-6 methodology relies on Monte Carlo simulation. It is well known that simulation models perform best when the key parameters are calibrated and validated to local conditions. An overview of a proposed calibration process is leveraged from Spiegelman et al. (15) and illustrated in Figure 10.



Figure 10. Proposed HCM calibration process. 
The first step is to collect appropriate empirical performance data on a number of arterial roadways that will be studied. Intuitively, the best data would be individual trajectory data that may be aggregated into average travel times for specific periods (e.g., $15 \mathrm{~min}$ ) that correspond to the HCM-6 output. In this paper, BT data were utilized. With the recent growth in vehicle-to-vehicle and vehicle-to-road infrastructure communications, there is great potential in using these technologies to obtain accurate point-to-point travel times on urban street facilities. The continuous development of the connectivity of communication devices is now improving on accuracy and cost-effectiveness for travel time research (7).

The second step is to model the arterials by using the STREETVAL engine. As discussed in this paper the primary output is the distribution of average travel times for the given study period. This information is used to develop the TTR metrics.

The third step is to compare the output from the STREETVAL engine with the empirical data. Based on experience the authors argue that the average travel time distributions should be analyzed statistically. This would involve a goodness of fit measure such as the $\mathrm{K}-\mathrm{S}$ test or chisquare test or some of the more typical nonparametric tests.

If the two distributions are not statistically different, then the process can be stopped, and the model can be considered calibrated. Note that it would be useful to validate the model using empirical data that were not part of the calibration process (15). It should also be noted that other output, such as mean travel time (MTT) or absolute percentage error, may also be used to ascertain how similar the travel time distributions are to each other.

Assuming that the simulated results and empirical data are dissimilar, the user may then change the STREETVAL parameters. The process is repeated until the results are acceptable or until a set number of iterations has been reached. The authors assume that this procedure will be automatic such that the optimization step utilizes standard techniques such as genetic algorithms or the Simplex Method (15).

The proposed calibration methodology may also be used to augment the HCM-6 reliability methodology for predicting TTR for other scenarios not currently included such as traffic signal malfunction, adverse weather conditions, and railroad crossing and preemption events (5). 


\section{More Robust Input Data}

As shown in Figure 10, there are four main input classes: supply, traffic, weather, and incidents. The current HCM methodology is designed to provide forecast TTR metrics in an input data-poor environment. However, there is the potential to allow for more disaggregate input data. For example, cities such as Lincoln have detailed weather data disaggregated by day of year and hour of day. These data may be directly correlated to the travel time data that were collected in this study and aggregated into 15-min averages for each day of the year. It would be useful if users could employ these data as input to STREETVAL. It is hypothesized that this would result in more accurate travel time distributions particularly if coupled with the calibration scheme proposed in Figure 10.

Similarly, it is possible in many cities to obtain general volume patterns, and incident/crash patterns at a fairly disaggregate level. This information could also be used as input and would potentially allow for more accurate results from the scenario degeneration process. It is hypothesized that if all four input data input streams were more disaggregate and were disaggregated at the same level (e.g., crash, volume, and weather data at 15-min intervals) the resulting travel time distribution from the Monte Carlo simulation study would be much more accurate.

In summary, two augmentation schemes have the potential to lead to more realistic travel time distributions and, ultimately, more accurate TTR metrics. Note that these two approaches are not mutually exclusive and it could be argued that using both approaches would be superior to using only one.

\section{Concluding Remarks}

The current and 6th edition of the HCM methodology for evaluating TTR on an urban street facility incorporates nonrecurring congestion effects including weather events, incident events, and work zones. The HCM-6 approach is based on a single day volume count within the year, and the volume for the remaining 364 days are estimated based on this volume, demand factors, predicted weather, and expected incident occurrences.

The objective of this paper was to evaluate the TTR of an urban street facility using low cost archived BT data. The goals were to validate the HCM-6 urban street reliability methodology by comparing the empirical BT travel time distributions with the estimated $\mathrm{HCM}-6$ distribution, and 
to propose potential HCM-6 augmentation strategies. The selected testbed was an urban street facility in Lincoln, NE, a city that has its weather data recommended as one of the default settings in the HCM- 6 computational engine. The PM peak travel times for all weekdays within a year were analyzed. The results from the BT analysis were compared to the outcome of the HCM-6 methodology. It was determined that:

1. The testbed MTTs differ by approximately $4 \mathrm{~s}$. This difference was found to be statistically significant at a 95\% confidence level. However, from a practical or engineering perspective, this difference is negligible.

2. The HCM-6 methodology resulted in travel time estimates that had considerably less variability than the empirical data. It was hypothesized that the differences occurred because of measurement uncertainty, highly aggregated input data, and the fact that the model was not calibrated to local conditions.

3. The HCM-6 methodology underestimated the buffer index and the planning time index by approximately $62 \%$ and $9 \%$, respectively. However, both the HCM- 6 results and the empirical BT results yielded a TTI that implied that the testbed is likely to provide more than $95 \%$ VMT associated with a LOS D or better. This was true because the predetermined LOS was at "C."

Two augmentation strategies for the HCM- 6 travel time forecasting methodology were proposed including calibrating the model to local conditions and allowing for more disaggregate input data to be used. In addition to studying these proposed augmentation strategies, it would be useful to examine the temporal and geographic transferability conditions associated with the HCM-6 model. It would also be useful to examine other test scenarios including snow days, lane closures, and work zone capacity reductions.

Acknowledgments - The authors thank John Sangster and Amber Hadenfeldt of the Nebraska Transportation Center for their assistance with data collection and edits. The authors thank the City of Lincoln, especially Andy Jenkins, for making testbed data readily available. The Standing Committee on Highway Capacity and Quality of Service (AHB40) peer-reviewed this paper (19-04239). The contents of this paper reflect the views of the authors, who are responsible for the facts and accuracy of the information presented herein and are not necessarily representative of the state of Nebraska or the City of Lincoln. 
Author contributions - The authors contributed to the paper as follows: study conception and design: $E T, L R$; data collection: $E T$; analysis and interpretation of results: ET, LR; and manuscript preparation: ET, LR. Both authors reviewed the findings and approved the final version of the manuscript.

\section{References}

1. Toppen, A., and K. Wunderlich. Travel Time Data Collection for Measurement of Advanced Traveler Information Systems Accuracy. No. FHWA-OP-03-183. Mitretek Systems, Falls Church, VA, 2003.

2. Travel Time Reliability: Making It There on Time, All the Time. Federal Highway Administration, US Department of Transportation, Office of Operations Report, 2006. https://ops.fhwa.dot.gov/publications/tt reliability/TTR Report.htm Accessed February 5, 2018.

3. Cambridge Systematics, Inc., Texas Transportation Institute, University of Washington, and Dowling Associates. Providing a Highway Systems with Reliable Travel Times: Study 3-Reliability. Final Report, NCHRP Project 20- 58(3). Transportation Research Board of the National Academies, Washington, D.C., 2003.

4. Highway Capacity Manual. A Guide for Multimodal Mobility Analysis. Transportation Research Board, Washington, D.C., 2016.

5. Zegeer, J., J. Bonneson, R. Dowling, and P. Ryus. Incorporating Travel Time Reliability into the Highway Capacity Manual. [Supporting Datasets]. No. SHRP 2 Report S2- L08-RW-1. United States. National Transportation Library [Distributor], Washington, D.C., 2013.

6. Samandar, M. S., B. M. Williams, and A. Wagner. Validation of Travel Time Reliability Prediction from Probe Data. No. Project ID: NTC2014-SU-R-22. National Transportation Center, University of Maryland, Baltimore, MD, 2018.

7. National Climatic Data Center. Comparative Climatic Data for the United States through 2010. National Oceanic and Atmospheric Administration, Asheville, NC, 2010. http://www.ncdc.noaa.gov

8. Hallenbeck, M., M. Rice, B. L. Smith, C. Cornell-Martinez, and J. Wilkinson. Vehicle Volume Distributions by Classification. No. FHWA-PL-97-025. Chaparral Systems Corporation, Federal Highway Administration, Washington, D.C., 1997.

9. Tufuor, E. O. A, and L. R. Rilett. Analysis of Low Cost Bluetooth-Plus-WiFi Device for Travel Time Research. No. 18-05992. Presented at 97th Annual Meeting of the Transportation Research Board, Washington, D.C., 2018.

10. Haghani, A., M. Hamedi, K. F. Sadabadi, S. Young, and P. Tarnoff. Data Collection of Freeway Travel Time Ground Truth with Bluetooth Sensors. Transportation Research Record: Journal of the Transportation Research Board, 2010. 2160: 60-68.

11. Brennan, T. M., Jr., J. M. Ernst, C. M. Day, D. M. Bullock, J. V. Krogmeier, and M. Martchouk. Influence of Vertical Sensor Placement on Data Collection Efficiency from Bluetooth MAC Address Collection Devices. Journal of Transportation Engineering, Vol. 136, 2010, pp. 1104-1109. 
12. Hainen, A. M., J. S. Wasson, S. M. L. Hubbard, S. M. Reimas, G. D. Farnsworth, and D. M. Bullock. Estimating Route Choice and Travel Time Reliability with Field Observations of Bluetooth Probe Vehicles. Transportation Research Record: Journal of the Transportation Research Board, 2011. 2256: 43-50.

13. Mahmassani, H. S., J. Kim, Y. Chen, and Y. Stogios. Incorporating Reliability Performance Measures into Operations and Planning Modeling Tools. Transportation Research Board of the National Academies, Washington, D.C., 2014.

14. Tarnoff, P. J., D. M. Bullock, S. E. Young, J. Wasson, N. Ganig, and J. R. Sturdevant. Continuing Evolution of Travel Time Data Information Collection and Processing. Presented at 88th Annual Meeting of the Transportation Research Board, Washington, D.C., 2009.

15. Spiegelman, C., E. S. Park, and L. R. Rilett. Transportation Statistics and Microsimulation. CRC Press, Boca Raton, FL, 2016. 